\title{
Disturbance Observer-Based Input-Output Finite-Time Control of a Class of Nonlinear Systems
}

\author{
Leipo Liu, Xiaona Song, Zhumu Fu, and Shuzhong Song \\ College of Information Engineering, Post-Doctor Station of Control Science and Engineering, \\ Henan University of Science and Technology, Luoyang 471003, China
}

Correspondence should be addressed to Leipo Liu; liuleipo123@163.com

Received 21 July 2016; Revised 1 November 2016; Accepted 2 August 2017; Published 6 September 2017

Academic Editor: Yuri Vladimirovich Mikhlin

Copyright (C) 2017 Leipo Liu et al. This is an open access article distributed under the Creative Commons Attribution License, which permits unrestricted use, distribution, and reproduction in any medium, provided the original work is properly cited.

This paper is concerned with disturbance observer-based input-output finite-time control of a class of nonlinear systems with onesided Lipschitz condition, as well as multiple disturbances. Firstly, a disturbance observer is constructed to estimate the disturbance generated by an exogenous system. Secondly, by integrating the estimation of disturbance with a classical state feedback control law, a composite control law is designed and sufficient conditions for input-output finite-time stability (IO-FTS) of the closed-loop system are attained. Such conditions can be converted into linear matrix inequalities (LMIs). Finally, two examples are given to show the effectiveness of the proposed method.

\section{Introduction}

The robust Lyapunov stability reflects the asymptotic behavior; that is, the result only is achieved in an infinite-time interval. However, in many practical applications (for example, in biochemical reaction systems, communication network systems, or robot control systems), one is more interested in what happens over a finite-time interval rather than the asymptotical property. To discuss this transient performance, Dorato [1] firstly defined finite-time stability (FTS) for linear deterministic systems. A system is said to be FTS if, given a bound on the initial condition, its state does not exceed a certain threshold during a specified time interval. Up until now, much work has been done in this field [2-4]. Recently, the definition of input-output finite-time stability (IO-FTS) has been firstly introduced in [5], which is a more practical concept and means that, given a class of norm-bounded input signals over a specified time interval of length $T$, the outputs of the system do not exceed an assigned threshold during such time interval. This definition of IO-FTS is fully consistent with the definition of FTS. IO-FTS involves signals defined over a finite-time interval and does not necessarily require the inputs and outputs to belong to the same class, and IO-FTS constraints permit specifying quantitative bounds on the controlled variables to be fulfilled during the transient response $[6,7]$. Some related results are also presented, such as linear systems [8], hybrid systems via static output feedback [9], nonlinear systems via sliding mode control [10], discrete-time impulsive switch systems [11], nonlinear stochastic systems [12], and Markovian jump systems [13, 14].

On the other hand, the complex systems include multiple disturbances, such as unknown frictions or loads, harmonic disturbances, modeling uncertainties, and stochastic noises. The presence of different types of disturbances will seriously affect control accuracy. Therefore, how to design a controller to suppress disturbances is a hot topic. So disturbance observer-based control technique is proposed as an effective approach, and many related meaningful results are presented [15-19]. It is worth noting that the most existing results involve the asymptotic stability and system nonlinearity functions are assumed to satisfy the Lipschitz condition. As we know, one-sided Lipschitz condition is shown to be an extension of the Lipschitz condition and is less conservative, and the one-sided Lipschitz constant is significantly smaller than the Lipschitz constant, which makes it much more suitable for estimating the influence of nonlinear part [2024]. Recently, [25] considers the finite-time control of nonlinear systems with one-sided Lipschitz condition. However, 
there are few results about disturbance observer-based inputoutput finite-time control of nonlinear systems with onesided Lipschitz condition, which motivates our study.

This paper considers disturbance observer-based inputoutput finite-time control of a class of nonlinear systems with one-sided Lipschitz condition, as well as disturbances. The system model includes two parts of disturbances. One part is a norm-bounded disturbance. The other part is supposed by an exogenous system, which is supposed to have a modeling perturbation. Firstly, a reduced-order disturbance observer is designed to estimate the disturbance generated by this exogenous system. Secondly, a composite control law is designed, which includes the estimation of disturbance and the state feedback control law. Moreover, sufficient conditions are derived to guarantee that the closed-loop system is IOFTS. Such conditions can be converted into linear matrix inequalities (LMIs). Finally, two examples are given to show the effectiveness of the proposed method.

Notations. In this paper, $R^{n}$ and $R^{n \times m}$ denote, respectively, the spaces of $n$-dimensional real numbers and $n \times m$ real matrices. Let $M$ be a real symmetric matrix; $M>0$ means $M$ is positive definite. $L_{2}$ stands for the space of square integrable vector functions. $\|\cdot\|$ refers to the Euclidean vector norm. * represents the omitted symmetric element of a matrix. $\langle\cdot, \cdot\rangle$ is the inner product in $R^{n}$; that is, given $x, y \in R^{n}$, then $\langle x, y\rangle=x^{T} y$, where $x^{T}$ is the transpose of the column vector $x \in R^{n}$.

\section{Problem Formulation}

Consider the following nonlinear system:

$$
\dot{x}(t)=A x(t)+B\left(u(t)+d_{1}(t)\right)+\phi(x)+D d_{2}(t),
$$

where $x(t) \in R^{n}$ is the state vector and $u(t) \in R^{m}$ is the control input. $d_{1}(t) \in R^{m}$ can represent the constant and the harmonic noises, which is described by an exogenous system in Assumption 4. $d_{2}(t) \in R^{q}$ is the external disturbance, which is assumed to be an arbitrary signal in $L_{2} \cdot \phi(x)$ represents a nonlinear function that is continuous with respect to $x(t)$ and $\phi(0)=0 . A, B, D$ are matrices with compatible dimensions and $(A, B)$ is controllable.

The following concepts about Lipschitz property, the onesided Lipschitz property, and quadratic inner-boundedness property for the nonlinear function $\phi(x)$ are introduced to further our study.

Definition 1. The nonlinear function $\phi$ is said to be locally Lipschitz in a region $Q$ including the origin with respect to $x$, if there exists a constant $l>0$ satisfying

$$
\left\|\phi\left(x_{1}\right)-\phi\left(x_{2}\right)\right\| \leq l\left\|x_{1}-x_{2}\right\|, \quad \forall x_{1}, x_{2} \in Q .
$$

Definition 2. The nonlinear function $\phi$ is said to be one-sided Lipschitz, if there exists a constant $\rho \in R$ such that

$$
\begin{aligned}
\left\langle\phi\left(x_{1}\right)-\phi\left(x_{2}\right), x_{1}-x_{2}\right\rangle \leq \rho\left\|x_{1}-x_{2}\right\|^{2} & \\
& \forall x_{1}, x_{2} \in Q,
\end{aligned}
$$

where $\rho$ is called the one-sided Lipschitz constant.
From Definitions 1 and 2, Lipschitz constant $l$ must be positive; however, one-sided Lipschitz constant $\rho$ can be positive, zero, or even negative. It is true that any Lipschitz function is also one-sided Lipschitz, not vice versa [24].

Definition 3. The nonlinear function $\phi$ is called quadratic inner-bounded in the region $\bar{Q}$, if there exist constants $\beta, \gamma \in$ $R$ such that

$$
\begin{aligned}
\Delta \phi^{T} \Delta \phi \leq \beta\left\|x_{1}-x_{2}\right\|^{2}+\gamma\left\langle x_{1}-x_{2}, \Delta \phi\right\rangle & \\
& \forall x_{1}, x_{2} \in \bar{Q}
\end{aligned}
$$

with $\Delta \phi=\phi\left(x_{1}\right)-\phi\left(x_{2}\right)$.

From the definition, any Lipschitz function is quadratically inner-bounded with $\beta>0$ and $\gamma=0$, but the converse is not true. Note that $\gamma$ is not necessarily positive. In fact, if $\gamma$ is restricted to be positive, then it can be shown that $\phi$ must be Lipschitz.

Assumption 4. The disturbance $d_{1}(t)$ in (1) can be described by

$$
\begin{aligned}
\dot{\varphi}(t) & =W \varphi(t)+M d_{3}(t), \\
d_{1}(t) & =V \varphi(t),
\end{aligned}
$$

where $W \in R^{r \times r}, M \in R^{r \times s}$, and $V \in R^{m \times r}$ are matrices with compatible dimensions. $d_{3}(t) \in R^{s}$ is the addition disturbance in $L_{2}$, which results from the perturbations and uncertainties in the exogenous system.

Remark 5. In (5), if $W=\left[\begin{array}{cc}0 & a \\ -a & 0\end{array}\right]$ with $a>0$ is held, then $d_{1}(t)$ represents the harmonic disturbance and $a$ denotes the frequency of the harmonic disturbance [19].

The disturbance observer is constructed as

$$
\begin{aligned}
\widehat{d}_{1}(t)= & V \widehat{\varphi}(t), \\
\widehat{\varphi}(t)= & v(t)-L x(t), \\
\dot{v}(t)= & (W+L B V)(v(t)-L x(t)) \\
& +L(A x(t)+\phi(x)+B u(t))
\end{aligned}
$$

and a feedback controller is designed as

$$
u(t)=-\widehat{d}_{1}(t)+K x(t)
$$

where the observer gain $L \in R^{r \times n}$ and the controller gain $K \in$ $R^{m \times n}$ will be designed later, respectively.

Let the estimation error be $e(t)=\varphi(t)-\widehat{\varphi}(t)$. From (1) and (5) $-(7)$, the error equation is

$$
\dot{e}(t)=(W+L B V) e(t)+L D d_{2}(t)+M d_{3}(t) .
$$


Denote $\bar{x}(t)=\left[\begin{array}{ll}x^{T}(t) & e^{T}(t)\end{array}\right]^{T}$ and $\bar{d}(t)=\left[\begin{array}{ll}d_{2}^{T}(t) & d_{3}^{T}(t)\end{array}\right]^{T}$. From (1) and (6)-(8), the resulting closed-loop system can be written in the form as follows:

$$
\begin{aligned}
\dot{\bar{x}}(t)= & {\left[\begin{array}{cc}
A+B K & B V \\
0 & W+L B V
\end{array}\right] \bar{x}(t)+\left[\begin{array}{l}
I \\
0
\end{array}\right] \phi(x) } \\
& +\left[\begin{array}{cc}
D & 0 \\
L D & M
\end{array}\right] \bar{d}(t) .
\end{aligned}
$$

The reference output is set as

$$
z(t)=C_{1} x(t)+C_{2} e(t)=C \bar{x}(t),
$$

where $C=\left[\begin{array}{ll}C_{1} & C_{2}\end{array}\right], C_{1} \in R^{p \times n}$, and $C_{2} \in R^{p \times r}$.

In this work, a class of norm-bounded square integrable signals $\bar{d}(t)$ over $[0, T]$ is defined as follows:

$$
\Omega=\left\{\bar{d}(\cdot) \in L_{2}: \int_{0}^{T} \bar{d}^{T}(t) S \bar{d}(t) d t \leq 1\right\},
$$

where $T>0$ and $S=\left[\begin{array}{cc}S_{11} & S_{12} \\ * & S_{22}\end{array}\right]>0$.

Definition 6 (IO-FTS). Given a time interval $[0, T]$, disturbance signals $\Omega$ defined by (11) and a weighted matrix $R>0$. The closed-loop system (9) is said to be IO-FTS with respect to $(T, \Omega, R)$, if for $\bar{x}(0)=0$,

$$
\bar{d}(t) \in \Omega \Longrightarrow z^{T}(t) R z(t) \leq 1, \quad \forall t \in[0, T] .
$$

Remark 7. In $[5,7]$, the authors have proposed two definitions of IO-FTS for two different classes of disturbance signals, respectively, that is, the norm-bounded square integrable signals $\left(\bar{d}(t)\right.$ satisfies $\left.\int_{0}^{T} \bar{d}^{T}(t) S \bar{d}(t) d t \leq 1\right)$ and the uniformly bounded signals $\left(\bar{d}(t)\right.$ satisfies $\left.\max _{t \in[0, T]} \bar{d}^{T}(t) S \bar{d}(t) d t \leq 1\right)$. Because of similar approach, we only focus on the former in Definition 6.

Remark 8. In (10), the reference output $z(t)$ includes the estimation error $e(t)$. From Definition 6, our goal is that the weighted system output $z^{T}(t) R z(t)$ does not exceed threshold 1 in a given time interval $T$; then the estimation error $e(t)$ might not converge to zero in a given time interval $T$. If a smaller threshold is chosen, then the estimation error will become very small.

\section{IO-FTS Analysis}

In this section, we will give some sufficient conditions for IOFTS of the closed-loop system (9).

Theorem 9. Given a scalar $\alpha>0$. Suppose the function $\phi(x)$ satisfies conditions (3) and (4) with constants $\rho, \beta$, and $\gamma$. If there exist matrices $P=\operatorname{diag}\left\{P_{1}, P_{2}\right\}>0, K, L$ and the scalars $\varepsilon_{1}>0$ and $\varepsilon_{2}>0$ such that the following nonlinear matrix inequalities are true:

$$
\begin{aligned}
& \Pi=\left[\begin{array}{cccc}
\Pi_{1} & P_{1} B V & \frac{\gamma \varepsilon_{2}-\varepsilon_{1}}{2} I+P_{1} & \Pi_{3} \\
* & \Pi_{2} & 0 & \Pi_{4} \\
* & * & -\varepsilon_{2} I & 0 \\
* & * & * & -S
\end{array}\right]<0, \\
& {\left[\begin{array}{cc}
-e^{-\alpha T} P & C^{T} \\
* & -R^{-1}
\end{array}\right]<0,}
\end{aligned}
$$

where $\Pi_{1}=P_{1}(A+B K)+(A+B K)^{T} P_{1}+\varepsilon_{1} \rho I+\varepsilon_{2} \beta I-\alpha P_{1}$, $\Pi_{2}=P_{2}(W+L B V)+(W+L B V)^{T} P_{2}-\alpha P_{2}, \Pi_{3}=\left[\begin{array}{ll}P_{1} D & 0\end{array}\right]$, and $\Pi_{4}=\left[\begin{array}{ll}P_{2} L D & P_{2} M\end{array}\right]$, then the closed-loop system (9) is IO-FTS with respect to $(T, \Omega, R)$.

Proof. Consider the following Lyapunov functional candidate:

$$
V(t)=\bar{x}^{T}(t) P \bar{x}(t),
$$

where $P=\operatorname{diag}\left\{P_{1}, P_{2}\right\}>0$.

The time derivative of $V(t)$ along the trajectories of system (10) is given by

$$
\begin{aligned}
\dot{V}(t) & \\
= & x^{T}(t)\left[P_{1}(A+B K)+(A+B K)^{T} P_{1}\right] x(t) \\
& +2 x^{T}(t) P_{1} B V e(t) \\
& +e^{T}(t)\left[P_{2}(W+L B V)+(W+L B V)^{T} P_{2}\right] e(t) \\
& +2 x^{T}(t) P_{1} \phi(x)+2 x^{T}(t) P_{1}\left[\begin{array}{ll}
D & 0
\end{array}\right] \bar{d}(t) \\
& +2 e^{T}(t) P_{2}\left[\begin{array}{ll}
L D & M
\end{array}\right] \bar{d}(t) .
\end{aligned}
$$

From (3), for any positive scalar $\varepsilon_{1}$, we have

$$
\varepsilon_{1}\left[\begin{array}{l}
x(t) \\
\phi(x)
\end{array}\right]^{T}\left[\begin{array}{cc}
\rho I & -\frac{1}{2} I \\
* & 0
\end{array}\right]\left[\begin{array}{l}
x(t) \\
\phi(x)
\end{array}\right] \geq 0 .
$$

From (4), similarly, for any positive scalar $\varepsilon_{2}$, we have

$$
\varepsilon_{2}\left[\begin{array}{l}
x(t) \\
\phi(x)
\end{array}\right]^{T}\left[\begin{array}{cc}
\beta I & \frac{\gamma}{2} I \\
* & -I
\end{array}\right]\left[\begin{array}{l}
x(t) \\
\phi(x)
\end{array}\right] \geq 0 .
$$

From (16) to (18), we have

$$
\dot{V}(t)-\alpha V(t)-\bar{d}^{T}(t) S \bar{d}(t) \leq \xi^{T}(t) \Pi \xi(t),
$$

where $\xi(t)=\left[\begin{array}{llll}x^{T}(t) & e^{T}(t) & \phi^{T}(x) & \bar{d}^{T}(t)\end{array}\right]^{T}$.

If (13) is held, then we have

$$
\dot{V}(t)<\alpha V(t)+\bar{d}^{T}(t) S \bar{d}(t) .
$$


Integrating (20) from 0 to $t$, with $t \in(0, T]$, and using (11), we obtain

$$
\begin{aligned}
V(t) & <e^{\alpha t} V(0)+\int_{0}^{t} e^{\alpha(t-s)} \bar{d}^{T}(s) S \bar{d}(s) d s \\
& <e^{\alpha T} V(0)+e^{\alpha T} \int_{0}^{T} \bar{d}^{T}(s) S \bar{d}(s) d s \\
& <e^{\alpha T} V(0)+e^{\alpha T} .
\end{aligned}
$$

Noting that $\bar{x}(0)=0$, from (14) and (21), we have

$$
\begin{aligned}
z^{T}(t) R z(t) & =\bar{x}^{T}(t) C^{T} R C \bar{x}(t) \leq e^{-\alpha T} \bar{x}^{T}(t) P \bar{x}(t) \\
& \leq 1 .
\end{aligned}
$$

The proof is completed.

Theorem 10. Given a scalar $\alpha>0$. Suppose the function $\phi(x)$ satisfies conditions (3) and (4) with constants $\rho$, $\beta$, and $\gamma$. If there exist matrices $X_{1}>0, P_{2}>0, Y, N$ and the scalars, $\varepsilon_{1}>$ $0, \varepsilon_{2}>0$ such that the following nonlinear matrix inequalities are true:

$$
\begin{gathered}
{\left[\begin{array}{cccccc}
\bar{\Pi}_{1} & B V & \frac{\gamma \varepsilon_{2}-\varepsilon_{1}}{2} X_{1}+I & D & 0 & \sqrt{\left|\varepsilon_{1} \rho+\varepsilon_{2} \beta\right|} X_{1} \\
* & \bar{\Pi}_{2} & 0 & N D & P_{2} M & 0 \\
* & * & -\varepsilon_{2} I & 0 & 0 & 0 \\
* & * & * & -S_{11} & -S_{12} & 0 \\
* & * & * & * & -S_{22} & 0 \\
* & * & * & * & * & -I
\end{array}\right]} \\
<0, \\
{\left[\begin{array}{ccc}
-e^{-\alpha T} X_{1} & 0 & X_{1} C_{1}^{T} \\
* & -e^{-\alpha T} P_{2} & C_{2}^{T} \\
* & * & -R^{-1}
\end{array}\right]<0,}
\end{gathered}
$$

where $\bar{\Pi}_{1}=A X_{1}+B Y+X_{1} A^{T}+Y^{T} B^{T}-\alpha X_{1}$ and $\bar{\Pi}_{2}=$ $P_{2} W+N B V+W^{T} P_{2}+V^{T} B^{T} N^{T}-\alpha P_{2}$, then the closed-loop system (9) is IO-FTS with respect to $(T, \Omega, R)$. Furthermore, $K=Y X_{1}^{-1}$ and $L=P_{2}^{-1} N$.

Proof. For (13), denote that $X_{1}=P_{1}^{-1}$. Left- and rightmultiplying both sides of (13) by $\operatorname{diag}\left\{X_{1}, I, I, I, I\right\}$, leftand right-multiplying both sides of (14) by $\operatorname{diag}\left\{X_{1}, I, I\right\}$, respectively, and using Shur's complement, we easily obtain (23) and (24).

For (23), it is a nonlinear matrix inequality, and there are no effective algorithms for solving $X_{1}, P_{2}, Y, N, \varepsilon_{1}$, and $\varepsilon_{2}$ simultaneously. If the parameters $\varepsilon_{1}$ and $\varepsilon_{2}$ are given in advance, then (23) is converted to an LMI. So we easily solve it by MATLAB LMI toolbox. The procedure for constructing the gains $K$ and $L$ is summarized as follows.
Step 1. For a given scalar $\alpha>0$, choose the parameters $\varepsilon_{1}$ and $\varepsilon_{2}$.

Step 2. Calculate the matrices $X_{1}, P_{2}, Y$, and $N$ by solving LMIs (23) and (24). If there exists no solution, then the procedure returns to Step 1.

Step 3. Obtain the gains $K=Y X_{1}^{-1}$ and $L=P_{2}^{-1} N$.

\section{The Examples}

In this section, two examples are given to illustrate the effectiveness of the proposed scheme.

Example 1. Consider system (1) with (5) and (10); the system parameters are given as follows:

$$
\begin{aligned}
& A=\left[\begin{array}{ccc}
1 & -11 & -11 \\
31 & -1 & 1 \\
1 & 1 & 9
\end{array}\right] \\
& B=\left[\begin{array}{c}
1 \\
-1 \\
-1
\end{array}\right] \\
& D=\left[\begin{array}{c}
-1 \\
0 \\
1
\end{array}\right], \\
& W=\left[\begin{array}{cc}
0 & 5 \\
-5 & 0
\end{array}\right], \\
& M=\left[\begin{array}{c}
1 \\
-1
\end{array}\right], \\
& V=\left[\begin{array}{ll}
2 & -1
\end{array}\right], \\
& d_{2}(t)=0.01 e^{-t}, \\
& d_{3}(t)=0.02 e^{-t}, \\
& C_{1}=\left[\begin{array}{lll}
1 & -1 & -1
\end{array}\right], \\
& C_{2}=\left[\begin{array}{ll}
-1 & 1
\end{array}\right], \\
& \phi(x(t))=\left[\begin{array}{c}
\sin x_{1}(t)-2 x_{1}(t) \\
-2 x_{2}(t)+\cos x_{2}(t) \\
\sin x_{3}(t)-2 x_{3}(t)
\end{array}\right] .
\end{aligned}
$$

It is shown that $\phi(x(t))$ satisfies conditions (3) and (4) with $\rho=-1, \beta=9$, and $\gamma=0$.

The matrix $S=\left[\begin{array}{ll}1 & 0 \\ 0 & 2\end{array}\right]$ is selected; condition (11) can be satisfied. 


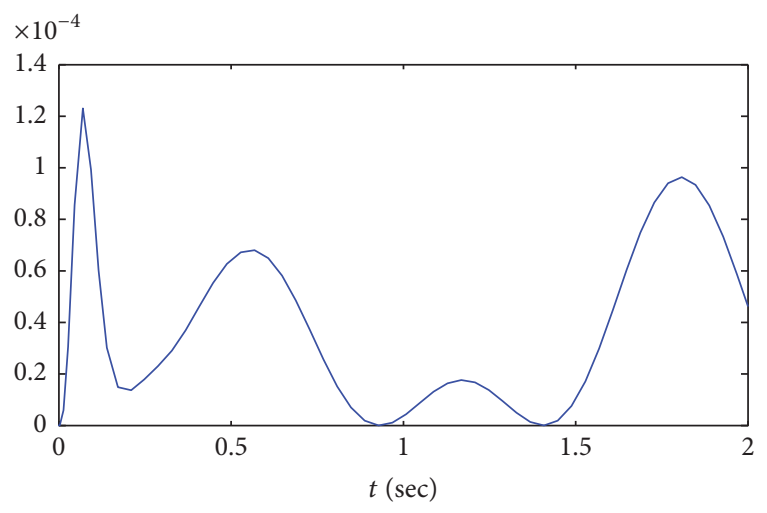

FIGURE 1: The weighted system output $z^{T}(t) R z(t)$.

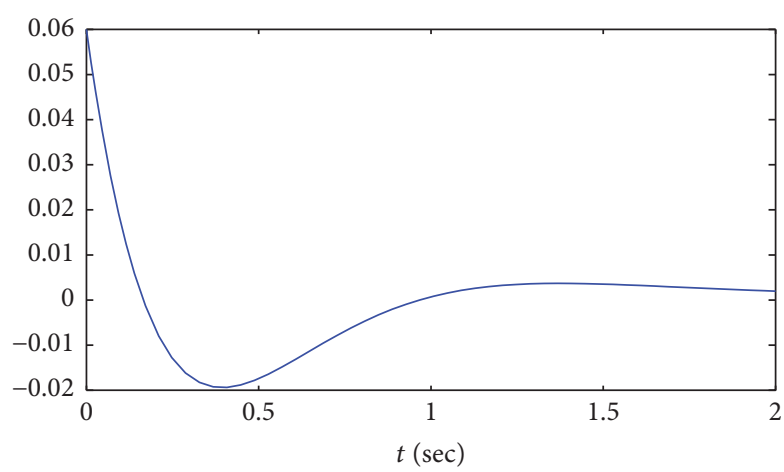

FIgURE 2: Disturbance estimation error $\left(d_{1}(t)-\widehat{d}_{1}(t)\right)$.

Choose $\alpha=0.001, \varepsilon_{1}=0.1, \varepsilon_{2}=1, R=0.03$, and $T=2$. Solving (23) and (24) yields

$$
\begin{aligned}
X_{1} & =\left[\begin{array}{ccc}
0.6432 & -0.4113 & -0.2118 \\
-0.4113 & 2.0671 & 1.0883 \\
-0.2118 & 1.0883 & 0.9475
\end{array}\right], \\
P_{2} & =\left[\begin{array}{cc}
3.6451 & -0.7529 \\
-0.7529 & 6.1927
\end{array}\right], \\
Y & =\left[\begin{array}{lll}
-16.7964 & 16.9650 & 24.6563
\end{array}\right], \\
N & =\left[\begin{array}{ccc}
-25.4743 & 9.7171 & -25.3660 \\
8.1713 & -13.3171 & 8.1096
\end{array}\right] .
\end{aligned}
$$

Therefore, we have

$$
\begin{aligned}
K & =\left[\begin{array}{lll}
-24.2668 & -18.8882 & 42.2949
\end{array}\right], \\
L & =\left[\begin{array}{ccc}
-6.8891 & 2.2789 & -6.8608 \\
0.4819 & -1.8734 & 0.4754
\end{array}\right] .
\end{aligned}
$$

The initial values $x(0), \varphi(0)$, and $v(0)$ are set as 0 . The simulation results are shown in Figures 1 and 2.

Figures 1 and 2 show the responses for weighted system output $z^{T}(t) R z(t)$ and disturbance estimation error $\left(d_{1}(t)-\right.$ $\left.\widehat{d}_{1}(t)\right)$, respectively. From the simulation results, we know
$z^{T}(t) R z(t)<1$; this implies the effectiveness of our proposed method.

Example 2. Consider system (1) with (5) and (10); the system parameters are given as follows:

$$
\begin{aligned}
& A=\left[\begin{array}{cc}
1 & 1 \\
-1 & -1
\end{array}\right] \text {, } \\
& B=\left[\begin{array}{c}
1 \\
-0.1
\end{array}\right] \text {, } \\
& D=\left[\begin{array}{c}
-1 \\
1
\end{array}\right] \\
& W=\left[\begin{array}{cc}
0 & 5 \\
-5 & 0
\end{array}\right] \text {, } \\
& M=\left[\begin{array}{c}
1 \\
-1
\end{array}\right] \text {, } \\
& V=\left[\begin{array}{ll}
2 & -1
\end{array}\right], \\
& d_{2}(t)=0.01 e^{-t}, \\
& d_{3}(t)=0.02 e^{-t}, \\
& C_{1}=\left[\begin{array}{ll}
1 & -1
\end{array}\right], \\
& C_{2}=\left[\begin{array}{ll}
-1 & 0
\end{array}\right] \text {, } \\
& \phi(x(t))=\left[\begin{array}{l}
-x_{1}(t)\left(x_{1}^{2}(t)+x_{2}^{2}(t)\right) \\
-x_{2}(t)\left(x_{1}^{2}(t)+x_{2}^{2}(t)\right)
\end{array}\right] .
\end{aligned}
$$

The above system model (1) can be used to describe the motion of a moving object [24]. It is shown that $\phi(x(t))$ does not satisfy condition (2). But it is one-sided Lipschitz with $\rho=0$.

Let

$$
\omega=\min \left(\sqrt{\frac{-\gamma}{4}}, \sqrt[4]{\beta+\frac{\gamma^{2}}{4}}\right), \quad \gamma<0, \beta+\frac{\gamma^{2}}{4}>0 .
$$

According to [16], the quadratically inner-bounded property of $\phi(x(t))$ is verified in $\bar{Q}, \bar{Q}=\left\{x \in R^{2}:\|x\| \leq \omega\right\}$. As the system is globally one-sided Lipschitz, that is, $Q=R^{2}, Q \cap$ $\bar{Q}=\bar{Q}$. Note that the region $\bar{Q}$ can be made arbitrarily large by choosing appropriate values for $\beta$ and $\gamma$. If the parameters $\beta=-0.01$ and $\gamma=-2$ are chosen, then $\omega=0.7071$.

The matrix $S=\left[\begin{array}{cc}10 & 0 \\ 0 & 100\end{array}\right]$ is selected; condition (11) can be satisfied.

Choose $\alpha=0.1, \varepsilon_{1}=0.1, \varepsilon_{2}=3, R=0.1$, and $T=5$. Solving (23) and (24) yields

$$
\begin{aligned}
& X_{1}=\left[\begin{array}{ll}
3.3269 & 0.0841 \\
0.0841 & 0.8640
\end{array}\right], \\
& P_{2}=10.3773,
\end{aligned}
$$




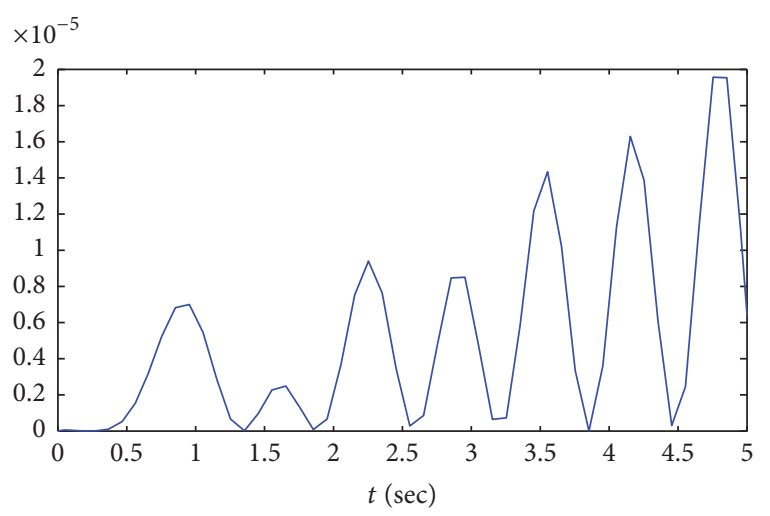

Figure 3: The weighted system output $z^{T}(t) R z(t)$.

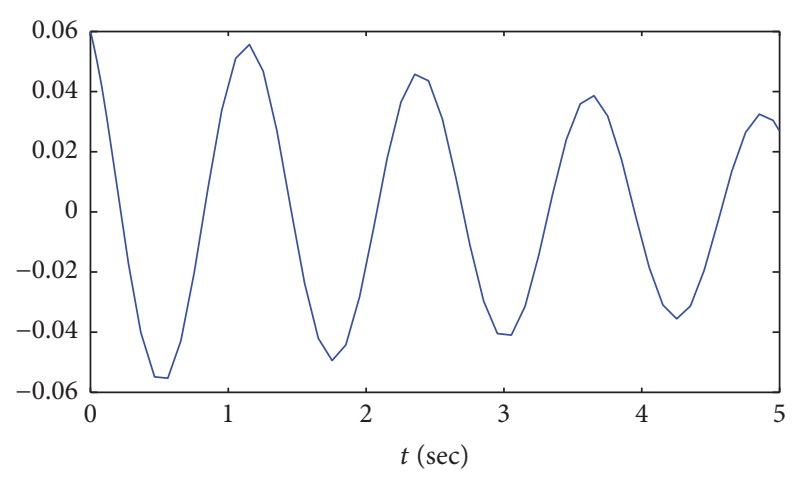

FIGURE 4: Disturbance estimation error $\left(d_{1}(t)-\widehat{d}_{1}(t)\right)$.

$$
\begin{aligned}
& Y=\left[\begin{array}{ll}
-89.2321 & 6.8977
\end{array}\right] \\
& N=-1.3750
\end{aligned}
$$

Therefore, we have

$$
\begin{aligned}
& K=\left[\begin{array}{ll}
-27.0897 & 10.6198
\end{array}\right] \\
& L=-0.1325
\end{aligned}
$$

The simulation results are shown in Figures 3 and 4.

Figures 3 and 4 show the responses for weighted system output $z^{T}(t) R z(t)$ and disturbance estimation error $\left(d_{1}(t)-\right.$ $\left.\widehat{d}_{1}(t)\right)$, respectively. From the simulation results, $z^{T}(t) R z(t)<$ 1 is held, so it is concluded that the proposed method is effective.

Remark 3. In Example 1, the nonlinear term $\phi(x(t))$ also satisfies the Lipschitz condition (2) with Lipschitz constant $l=3$. On the other hand, it also satisfies conditions (3) and (4) with $\rho=-1, \beta=9$, and $\gamma=0$. Obviously, the onesided Lipschitz constant $\rho=-1$ is smaller than the Lipschitz constant. In Example 2, $\phi(x(t))$ only satisfies the one-sided Lipschitz condition. From Examples 1 and $2, z^{T}(t) R z(t)<1$ can be held; that is, the weighted system output does not exceed an assigned threshold 1 during given time intervals, respectively.

\section{Conclusion}

This paper investigates the problem of disturbance observerbased input-output finite-time control of a class of nonlinear systems with one-sided Lipschitz condition, as well as multiple disturbances. Firstly, a reduced-order disturbance observer is designed to estimate the disturbance generated by this exogenous system. Secondly, by integrating the estimation of disturbance with the classical state feedback control law, a composite control law is designed to guarantee that the closed-loop system is IO-FTS. The obtained sufficient conditions can be converted into linear matrix inequalities (LMIs). Finally, two examples are given to show the effectiveness of the proposed method.

\section{Conflicts of Interest}

The authors declare that there are no conflicts of interest regarding the publication of this paper.

\section{Acknowledgments}

The authors are grateful for the support of the National Natural Science Foundation of China under Grants U1404610, 61473115 , and 61374077.

\section{References}

[1] P. Dorato, "Short time stability in linear time-varying systems," in Proceedings of the IRE International Convention Record Part 4, pp. 83-87, 1961.

[2] F. Amato, M. Ariola, and P. Dorato, "Finite-time control of linear systems subject to parametric uncertainties and disturbances," Automatica, vol. 37, no. 9, pp. 1459-1463, 2001.

[3] F. Amato, C. Cosentino, and A. Merola, "Sufficient conditions for finite-time stability and stabilization of nonlinear quadratic systems," IEEE Transactions on Automatic Control, vol. 55, no. 2, pp. 430-434, 2010.

[4] F. Amato, G. De Tommasi, and A. Pironti, "Necessary and sufficient conditions for finite-time stability of impulsive dynamical linear systems," Automatica, vol. 49, no. 8, pp. 2546-2550, 2013.

[5] F. Amato, R. Ambrosino, C. Cosentino, and G. De Tommasi, "Input-output finite time stabilization of linear systems," Automatica, vol. 46, no. 9, pp. 1558-1562, 2010.

[6] F. Amato, G. Carannante, and G. De Tommasi, "Input-output finite-time stability of switching systems with uncertainties on the resetting times," in Proceedings of the 19th Mediterranean Conference on Control and Automation (MED '11), pp. 13551360, Corfu, Greece, June 2011.

[7] F. Amato, G. Carannante, G. De Tommasi, and A. Pironti, "Input-output finite-time stability of linear systems: necessary and sufficient conditions," IEEE Transactions on Automatic Control, vol. 57, no. 12, pp. 3051-3063, 2012.

[8] Y. Guo, Y. Yao, S. Wang, K. Ma, K. Liu, and J. Guo, "Inputoutput finite-time stabilization of linear systems with finitetime boundedness," ISA Transactions, vol. 53, no. 4, pp. 977-982, 2014.

[9] F. Amato, G. Carannante, and G. De Tommasi, "Input-output finite-time stabilisation of a class of hybrid systems via static 
output feedback," International Journal of Control, vol. 84, no. 6, pp. 1055-1066, 2011.

[10] J. Song, Y. Niu, and Y. Zou, "Finite-time sliding mode control synthesis under explicit output constraint," Automatica, vol. 65, pp. 111-114, 2016.

[11] S. Huang, Z. Xiang, and H. R. Karimi, "Input-output finite-time stability of discrete-time impulsive switched linear systems with state delays," Circuits, Systems, and Signal Processing, vol. 33, no. 1, pp. 141-158, 2014.

[12] J. Song, Y. Niu, and T. Jia, "Input-output finite-time stabilisation of nonlinear stochastic system with missing measurements," International Journal of Systems Science, vol. 47, no. 12, pp. 29852995, 2016.

[13] Z. Tang and F. Liu, "Input-output finite-time stabilization of Markovian jump systems with convex polytopic switching probabilities," Journal of the Franklin Institute. Engineering and Applied Mathematics, vol. 353, no. 14, pp. 3632-3640, 2016.

[14] H. Ma and Y. Jia, "Input-output finite-time mean square stabilisation of stochastic systems with Markovian jump," International Journal of Systems Science, vol. 45, no. 3, pp. 325-336, 2014.

[15] X.-J. Wei, Z.-J. Wu, and H. R. Karimi, "Disturbance observerbased disturbance attenuation control for a class of stochastic systems," Automatica, vol. 63, pp. 21-25, 2016.

[16] X. Wei and N. Chen, "Composite hierarchical anti-disturbance control for nonlinear systems with DOBC and fuzzy control," International Journal of Robust and Nonlinear Control, vol. 24, no. 2, pp. 362-373, 2014.

[17] X. Wei, N. Chen, C. Deng, X. Liu, and M. Tang, "Composite stratified anti-disturbance control for a class of MIMO discretetime systems with nonlinearity," International Journal of Robust and Nonlinear Control, vol. 22, no. 4, pp. 453-472, 2012.

[18] X. Yao and L. Guo, "Composite anti-disturbance control for Markovian jump nonlinear systems via disturbance observer," Automatica, vol. 49, no. 8, pp. 2538-2545, 2013.

[19] L. Guo and S. Cao, Anti-Disturbance Control for Systems with Multiple Disturbances, CRC Press, Boca Raton, Flo, USA, 2013.

[20] W. Zhang, H. Su, F. Zhu, and D. Yue, "A note on observers for discrete-time lipschitz nonlinear systems," IEEE Transactions on Circuits and Systems II: Express Briefs, vol. 59, no. 2, pp. 123-127, 2012.

[21] M. Xu, G.-D. Hu, and Y. Zhao, "Reduced-order observer design for one-sided Lipschitz non-linear systems," IMA Journal of Mathematical Control and Information, vol. 26, no. 3, pp. 299317, 2009.

[22] Y. Zhao, J. Tao, and N.-Z. Shi, "A note on observer design for one-sided Lipschitz nonlinear systems," Systems \& Control Letters, vol. 59, no. 1, pp. 66-71, 2010.

[23] W. Zhang, H. Su, Y. Liang, and Z. Han, "Non-linear observer design for one-sided Lipschitz systems: an linear matrix inequality approach," IET Control Theory \& Applications, vol. 6, no. 9, pp. 1297-1303, 2012.

[24] W. Zhang, H. Su, H. Wang, and Z. Han, "Full-order and reduced-order observers for one-sided Lipschitz nonlinear systems using Riccati equations," Communications in Nonlinear Science and Numerical Simulation, vol. 17, no. 12, pp. 4968-4977, 2012.

[25] J. Song and S. He, "Robust finite-time Ho control for onesided Lipschitz nonlinear systems via state feedback and output feedback," Journal of the Franklin Institute. Engineering and Applied Mathematics, vol. 352, no. 8, pp. 3250-3266, 2015. 


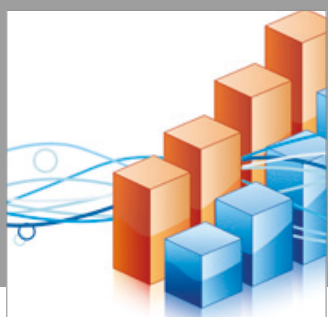

Advances in

Operations Research

vatersals

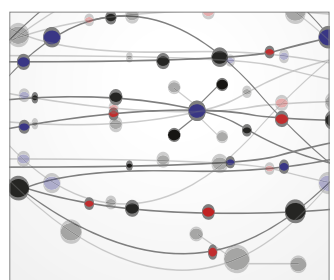

\section{The Scientific} World Journal
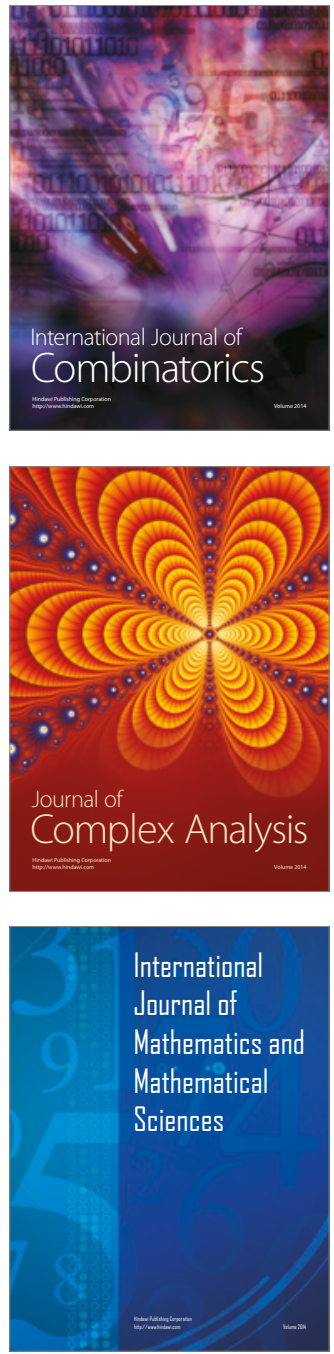
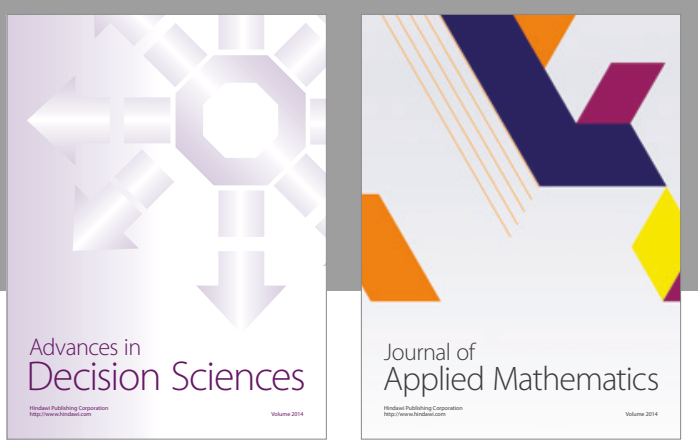

Algebra

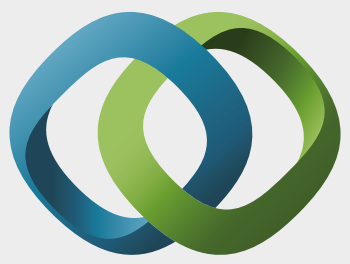

\section{Hindawi}

Submit your manuscripts at

https://www.hindawi.com
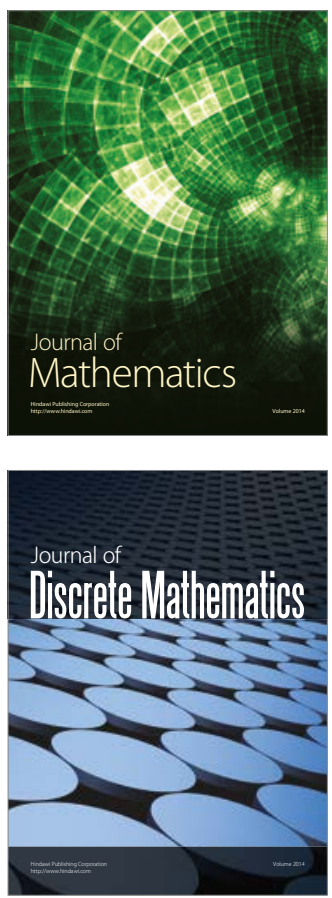

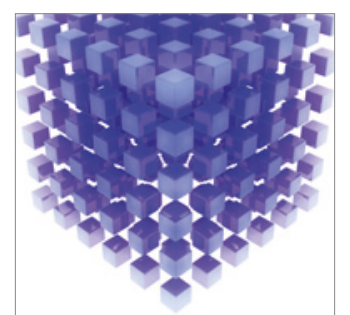

Mathematical Problems in Engineering
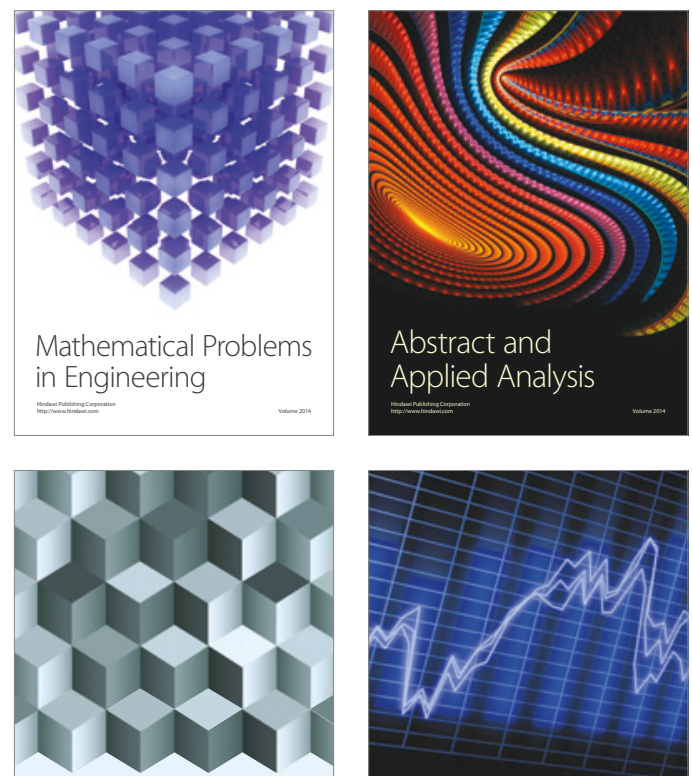

Journal of

Function Spaces

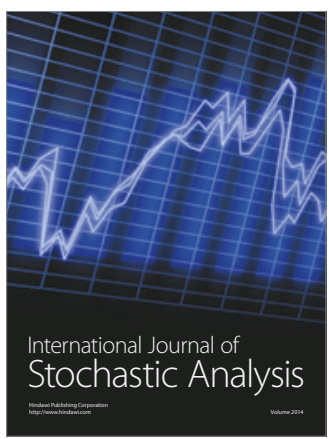

Probability and Statistics
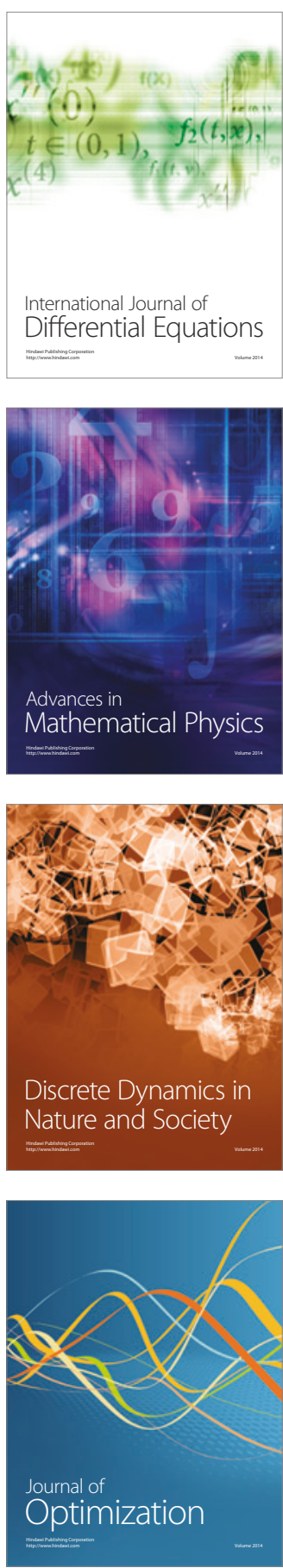